$\mathrm{ACI}$

17,1

90

Received 21 February 2018

Revised 1 July 2018

Accepted 2 July 2018

\section{Pricing schemes for air traffic services through multi-level approaches}

\author{
Rabah Guettaf
}

Department of Mathematics, Faculty of Sciences, University Mouloud Mammeri of Tizi Ouzou, Tizi Ouzou, Algeria, and

Félix Mora-Camino

Institute of Systems Science, Durban University of Technology, Durban, South Africa

\begin{abstract}
This article addresses the problem of air traffic service (ATS) pricing over a domestic air transportation system with either private or public ATS providers. In both cases, to take into account feedback effects on the air transportation market, it is considered that the adopted pricing approaches can be formulated through optimization problems where an imbedded optimization problem is concerned with the supply of air transportation (offered seat capacity and tariffs for each connection). Under mild assumptions in both situations the whole problem can be reformulated as a mathematical program with linear objective function and quadratic constraints. A numerical application is performed to compare both pricing schemes when different levels of taxes are applied to air carriers and passengers.
\end{abstract}

Keywords Quadratic optimization, Flows optimization in networks, Pricing, Air traffic services Paper type Original Article

\section{Introduction}

During the last decades, many studies in the field of Operations Research have been dedicated to the air transport sector by considering problems of planning, operation and pricing. In general, these studies consider the immediate effects of the decisions without taking into account indirect effects such as feedbacks which can be set up between the various actors of air transport. This has led to strategies which on the long term revealed to be largely suboptimal due to the unexpected reaction of other involved economic agents [1,2]. Thus, in this study a global approach including air traffic control, airports, airlines and passengers is developed for the pricing of the air navigation services as well as airport services considered as a whole as Air Traffic Services (ATS). The main ATS charges (en-route, approach and aerodrome charges) are collected for both air navigation and airport services. These different charges may also include provisions to reduce nuisances in the vicinity of airports as well as

\footnotetext{
(c) Rabah Guettaf and Félix Mora-Camino. Published in Applied Computing and Informatics. Published by Emerald Publishing Limited. This article is published under the Creative Commons Attribution (CCBY 4.0) license. Anyone may reproduce, distribute, translate and create derivative works of this article (for both commercial and non-commercial purposes), subject to full attribution to the original publication and authors. The full terms of this license may be seen at $\mathrm{http} / /$ /creativecommons.org/licences/by/4.0/legalcode

Publishers note: The publisher wishes to inform readers that the article "Pricing schemes for air traffic services through multi-level approaches" was originally published by the previous publisher of Applied Computing and Informatics and the pagination of this article has been subsequently changed. There has been no change to the content of the article. This change was necessary for the journal to transition from the previous publisher to the new one. The publisher sincerely apologises for any inconvenience caused. To access and cite this article, please use Guettaf, R., Mora-Camino, F. (2021), "Pricing schemes for air traffic services through multi-level approaches", Applied Computing and Informatics. Vol. 17 No. 1, pp. 90-107. The original publication date for this paper was 19/07/2018.
} 
other environmental impacts. These charges have been established in general on an empirical basis to cover broadly ATS costs without taking into consideration the reaction of the different actors of air transportation (Figure 1).

Here, two new pricing mechanisms are developed according to some fundamental assumptions:

- the main objective of airlines when defining their air transportation supply is to maximize their profit over an annual exercise;

- the main objective of a public ATS provider is to promote air transportation measured in flows of transported passengers while covering their operations costs;

- the main objective of a private ATS provider is to maximize his own profit while taking into account the profitability of the airline sector.

The revenue of the airlines depends directly of the price of their air tickets rates which should cover their operating costs which includes beyond fuel, crew and maintenance costs, their ATS charges. These ATS charges represent today between $10 \%$ and $20 \%$ of the operational costs of airlines. Thus, the pricing of ATS services has a global influence on ticket prices and affects the levels of demand for air transport (transport of passengers and freight).

In this study is considered the frequent case of a domestic air traffic area, presenting some international connections, being under the control of a single ATC provider. In general, air cargo and business aviation represent small shares of air transportation with activity levels rather inelastic with respect to the variation of ATC charges, so the focus is here on commercial flights operated by local airlines to transport passengers through the domestic network of air routes linking the different airports.

The cases where ATS operators are private or public are analyzed and particularized in this study. In both cases it seems essential to take into account the profit maximization behavior of the airlines when dimensioning their air transportation service levels (flights with associated frequencies and capacities) and setting their pricing policy. In order to limit the complexity of this study, the airlines sectors are considered as a whole without taking into account competition among them. This leads to the formulation of two-levels nonlinear optimization problems [3-5] which can be treated using already well established bi-level programming techniques [6-8].

For many high traffic airspaces, ATS pricing has been considered a potential tool to cope with saturation [9]. Recently, research studies considering the modulation of air navigation charges to cope with capacity and peak-loading pricing through a bi-level approach have been published, [10-12], where short term feedback effects between the ATS pricing and the airlines demand for ATS services are analyzed. Using a general bi-level pricing scheme [13], other studies have introduced the network dimension of ATS pricing, [14,15].

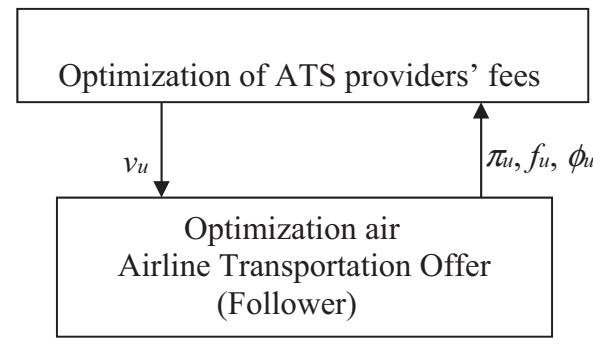

Figure 1. Two-level DecisionMaking Process. 
In the present study, the adopted long term analysis allows to introduce a third decision level which takes into account the final users (i.e. the passengers) reaction to ATS charges through their demand function.

\section{Current pricing practice for ATC/ATM}

Air navigation services (ATC/ATS service providers) finance in general their activities by charging airlines using their airspace. OACI publishes periodically updated general guidelines for pricing, [16]. The air navigation charges represent a significant portion of the cost of a flight for an airline [17], which has to increase ticket prices to cover them. These charges often represent between $10 \%$ and $20 \%$ of the cost of a flight, [18]. The Chicago Convention of 1947 which founded ICAO, has given the basis of the current charging systems for air navigation services. A detailed formula for the calculation of air traffic charges was not proposed at that time, but it was recommended to the states to establish a method to calculate the amount of charges to cover the costs of using specialized manpower and equipment (computers, radars and communication systems) to ensure the safety of air traffic. Different charges are collected today for air navigation (route, approach and aerodrome charges) and other airport services.

In the case of Europe, in the context of the Single European Sky operation [19], the central office for en-route charges (CRCO) of Eurocontrol is in charge of computing and collecting the charges paid by airspace users and of reassigning them to member countries traffic services. The following empirical formula has been used to compute the charges $R_{i}$ received by each state from a given flight:

$$
R_{i}=T_{i} \frac{D_{i}}{100} \times \sqrt{\frac{M}{50}}
$$

where $n$ is the number of considered states, $T_{i}$ is the unit rate adopted by state $i, D_{\mathrm{i}}$ is the distance flown in kilometers by that flight in the airspace of the state $i$, and $M$ is the maximum take-off weight in tons of the aircraft used in that flight. This unit rate varies in European countries from 22 (Ireland) to 90 (Belgium) Euros.

In United States, the Federal budget covers all operations and investment costs related with ATS/ATM since there are today no effective fees or charges for the users of the US airspace. The airspace and its resources are free for any plane of any size that conforms to the Federal Administration rules [20]. However, the air tickets comprise a set of taxes related with this use and which are collected by airlines. The exception is with flights that transit the US controlled airspace without departing or landing in United States. In that case the overflight charges consider different rates for the en-route and oceanic components of a flight. Different rates expressed per 100 nautical miles measured along the great circle distance between the entry and the exit points in the US-controlled airspace are applied. The charges are calculated with the formula which does not consider the mass or size of the aircraft:

$$
R_{i j}=r_{E} * D E_{i j} / 100+r_{O} * O D_{i j} / 100
$$

where $R i j$ is the total fee charged to aircraft flying between entry point $i$ and exit point $j$, $D E_{i j}$ is the total distance flown through each segment of en-route airspace between entry point $i$ and exit point $j, D O_{i i}$ is the total distance flown through each segment of oceanic airspace between entry point $i$ and exit point $j, r_{E}$ and $r_{O}$ are the en-route and oceanic rates, respectively around 60 and $25 \mathrm{US} \$$. The FAA review these rates at least once every two years and adjust them to reflect the current cost and volume of the services provided.

With the perspective of privatization of air navigation services in United States, a system of charges should be implemented for all users of the US airspace. Given the monopolistic nature of air navigation services, its charges should be regulated in order to avoid unfair pricing and to allow aviation users to pay the cost of the services to the air navigation service provider. 
From the above it appears that the current practice to establish charges for air navigation services does not take into account important factors related with the offer and the demand for air transportation:

- the structure of the operated air traffic network as a whole or with respect to each airline,

- the influence of air navigation charges on ticket fares and the level and structure of demand.

To take into account these two factors, in this study it is considered that the pricing approach adopted by public ATS providers can be formulated as an optimization problem through a bilevel optimization structure. This approach allows the consideration of the interactions between the different economic agents involved in air transportation. Then, reactive levels for supply by the airline companies and for demand by users as a result of the variation of ATS charges and air tickets prices can be taken into account when defining the air navigation charges.

\section{Definitions and assumptions}

Let $U$ be the set of local connections and let $E$ be the set of international connections. Let $\pi_{u}$ be the mean price for a seat on local connection $u \in U$ and let $\pi_{e}$ be the mean price of a seat on an international connectione $\in E$. Then the potential demands for local connections $u \in U$ are supposed to be given by $d_{u}=D_{u}(\underline{\pi}, \underline{f})$ where $f_{u}$ is the annual flow of aircraft on local connection $u$. Here $D_{u}$ is a demand function which is supposed to be of class $C^{\infty}$ from $\left(R^{+}\right)^{2|U|}$ to $R^{+}$where marginal variations of frequency and prices parameters are such that:

$$
\begin{aligned}
& \text { for } u \in U: \partial D_{u} / \partial \pi_{u} \leq 0, \text { for } v \in U, v \neq u: \partial D_{u} / \partial \pi_{v} \geq 0 \text { and }\left|\partial D_{u} / \partial \pi_{u}\right|>>\partial D_{u} / \partial \pi_{v} \\
& \text { for } \left.u \in U: \partial D_{u} / \partial f_{u} \geq 0, \text { for } v \in U, v \neq u: \partial D_{u} / \partial f_{v} \leq 0 \text { and } \partial D_{u} / \partial f_{u}>>\left|\partial D_{u} / \partial f_{v}\right|\right)
\end{aligned}
$$

The potential demands on international connections $e \in E$ are supposed to be given by $d_{e}=D_{e}\left(\pi_{e}, f_{e}\right)$ where $D_{e}$ is supposed to be a $C^{\infty}$ function from $\left(R^{+}\right)^{2}$ to $R^{+}$where:

$$
\partial D_{e} / \partial \pi_{e} \leq 0 \text { and } \partial D_{e} / \partial f_{e} \geq 0
$$

It is also supposed that the fields $\left(D_{u}, u \in U\right)$ and $\left(D_{e}, e \in E\right)$ are invertible with respect to $\left(\pi_{u}, u \in U\right)$ and $\left(\pi_{e}, e \in E\right)$. Let $\phi_{u}$ be the satisfied demand for local connection $u \in U$ and let $\phi_{e}$ be the satisfied demand on international connection $e \in E$, they should meet capacity and potential demand constraints given in equation (3):

$$
\phi_{u} \leq \min \left\{K_{u} \cdot f_{u}, D_{u}(\underline{\pi}, f)\right\} \text { and } \phi_{e} \leq \min \left\{K_{e} \cdot f_{e}, D_{e}\left(\pi_{e}, f\right)_{e}\right\}
$$

where $K_{u}$ is the mean seat capacity of flights on local connection $u, f_{e}$ is the annual flow of aircraft and $K_{e}$ is the mean seat capacity of flights on international connection $e$.

Let us define here different parameters to allow the quantification of the annual revenue of the ATS provider and the airline sector:

- Let $v_{u}$ be the ATS fee applied on a flight operating the local connection $u \in U$ and $v_{e}$ be the ATS fee applied on flights operating the international connections $e \in E$.

- Let $C_{A T S}^{F}$ be the fixed cost associated with ATS in the considered area, $\sigma_{u}$ be the variable average cost associated with ATS for a local connection $u \in U$ and $\sigma_{e}$ be the variable average cost associated with ATS for an international connectione $\in E$.

- Let $\lambda_{U}$ be the tax rate applied to the users of air transport on local connection $u \in U$ and let $\alpha_{\text {int }}$ be the part of this tax transferred to the ATS providers. 
$\mathrm{ACI}$

17,1

94

- Let $\lambda_{E}$ be the tax rate applied to the users of international flights along $e \in E$ and $\alpha_{e x t}$ be the part of this tax transferred to the ATS providers.

The annual economic return for the ATS operators, $R_{A T S}$, is then given by:

$$
R_{A T S}=\sum_{u \in U}\left(\left(v_{u}-\sigma_{u}\right) f_{u}+\alpha_{\mathrm{int}} \lambda_{U} \phi_{u} \pi_{u}\right)+\sum_{e \in E}\left(\left(v_{e}-\sigma_{e}\right) f_{e}+\alpha_{e x t} \lambda_{E} \phi_{e} \pi_{e}\right)-C_{A T S}^{F}
$$

Let $C_{A L S}^{F}$ be the fixed cost of the airlines sector operating $U$ and a part of $E$, let $c_{u}$ be the average operating cost for a flight along connection $u \in U$ and $c_{e}$ be the average operating cost for a flight along connectione $\in E$. Then, the annual economic return for the airlines sector, $R_{A L S}$, is given by:

$$
R_{A L S}=\sum_{u \in U}\left(\pi_{u}\left(1-\lambda_{U}\right) \phi_{u}-\left(c_{u}+v_{u}\right) f_{u}\right)+\mu \cdot \sum_{e \in E}\left(\pi_{e}\left(1-\lambda_{E}\right) \phi_{e}-\left(c_{e}+v_{e}\right) f_{e}\right)-C_{A L S}^{F}
$$

where $\mu \in[0,1]$ is the proportion of international traffic operated by local airlines. Given the total fleet of aircraft, the adopted theoretical fleet capacity for network $U, F_{U}$, is such as for any frequency distribution $\left\{f_{u}, u \in U\right\}$ :

$$
\sum_{u \in U} L_{u} f_{u} \leq F_{U}
$$

where $L_{u}$ is the block time associated to connection $u \in U$.

\section{ATS pricing through multi-level approach}

In this study it is considered that the definition of ATS charges must take into account the reaction of the airlines sector since these charges constitute a noticeable part of their operational costs. In the case of a public ATS provider, the final objective when fixing ATS charges is supposed to be the maximization of the total volume of passenger flows while considering the maximizing profit behavior of the airline sector and insuring budget equilibrium for the ATS provider. The main objective of a private ATS provider is in general to maximize its profit while providing acceptable conditions to the airlines sector to continue or develop its air transport activity. Here it is supposed that international flights $f_{e} \in E$ are fixed by international agreements while $\pi_{e} \in E$ are fixed by the international market. Once the ATS fees $\left(\nu_{u}, u \in U, \nu_{e}, e \in E\right)$ have been fixed, the airlines sector is supposed to fix his air transport supply to solve the following domestic problem with respect to $\left\{\left(\phi_{u}, \pi_{u}, f_{u}\right), u \in U\right\}$ :

$$
\max _{\phi_{u}, \pi_{u}, f_{u}} \sum_{u \in U}\left(\pi_{u} \phi_{u}-\left(\left(c_{u}+\nu_{u}\right) f_{u}\right)\right.
$$

with

$$
\begin{aligned}
& 0 \leq \phi_{u} \leq K_{u} \cdot f_{u} \quad \phi_{u} \leq D_{u}(\underline{\pi}, \underline{f}) u \in U \\
& \sum_{u \in U_{i}} L_{u} f_{u} \leq F_{U}
\end{aligned}
$$

and

$$
\pi_{u} \geq 0, f_{u} \geq 0 u \in U
$$

Let $\phi^{*}(\underline{\nu}), \underline{\pi}^{*}(\nu), f^{*}(\nu)$ be the solution of the above problem, then this solution will be profitable for the airline sector if: 


$$
\sum_{u \in U}\left(\pi_{u}^{*}\left(1-\lambda_{U}\right) \phi_{u}^{*}-\left(c_{u}+v_{u}\right) f_{u}^{*}\right)+\mu \cdot \sum_{e \in E}\left(\pi_{e}\left(1-\lambda_{E}\right) \phi_{e}-\left(c_{e}+v_{e}\right) f_{e}\right)-C_{A L S}^{F} \geq 0
$$

In the case of a public ATS provider, the problem of optimization of the pricing of ATS can be formulated in the following way:

$$
\max _{v_{u}, u \in U} \sum_{u \in U} \phi_{u}(\underline{\nu})+\sum_{e \in E} \phi_{e}
$$

with:

and

$$
\begin{gathered}
R_{A T S}\left(\underline{\phi}^{*}(\underline{\nu}), \underline{f}^{*}(\underline{\nu}), \underline{v}\right) \geq R_{A T S}^{\min } \\
R_{A L S}\left(\underline{\pi}^{*}(\underline{\nu}), \underline{f}^{*}(\underline{\nu}), \underline{\phi}^{*}(\underline{\nu}), \underline{\nu}\right) \geq R_{A L S}^{\min } \\
\nu_{u} \geq 0 \quad u \in U
\end{gathered}
$$

where $R_{A T S}^{\min }$ is the minimum acceptable economic return for the ATS operator and $R_{A L S}^{\min }$ is the minimum acceptable economic result for the airlines sector.

In this study, constraints on the economic result of the international airline companies are not considered. It is also supposed that ATS/ATM costs remain lower than a certain percentage of the revenue on an international connection:

$$
v_{e} f_{e} \leq \eta_{e} \phi_{e} \pi_{e} \text { with } 0<\eta_{e}<1 e \in E
$$

In the case of a private ATS provider, the problem of optimization of the pricing of ATS can be formulated in the following way:

$$
\max _{v_{u}, u \in U} R_{A T S}\left(\underline{\phi}^{*}(\underline{\nu}), \underline{f}^{*}(\underline{\nu}), \underline{v}\right)
$$

with:

$$
R_{A L S}\left(\underline{\pi}^{*}(\underline{\nu}), \underline{f}^{*}(\underline{\nu}), \underline{\phi}^{*}(\underline{\nu}), \underline{\nu}\right) \geq R_{A L S}^{\min }
$$

and

$$
\nu_{u} \geq 0 \quad u \in U
$$

where $R_{A L S}^{\min }$ is the minimum acceptable economic result for the airlines sector. In this case, $v_{e}, e \in E$ are such as:

$$
v_{e} f_{e}=\eta_{e} \phi_{e} \pi_{e} \quad \text { with } 0<\eta_{e}<1 e \in E
$$

In both cases, the ATS pricing problem configure a bi-level optimization problem, where the leader is the ATS supplier and the follower is the airlines sector. This leads to the two-level scheme:

\section{Air transport supply optimization by the airlines sector}

If it is supposed that on each link supply is not chosen superior to potential demand, conditions (8) can be rewritten as:

$$
\phi_{u}=K_{u} \cdot f \text { and } K_{u} \cdot f_{u} \leq D_{u}(\underline{\pi}, \underline{f}) u \in U
$$


since any overcapacity over a link will be an additional cost for the airlines and then the effective transported flow along link $u$ will be equal to the offered capacity on that link. Then the airlines sector problem (7)-(10) can be rewritten as:

$$
\max _{\pi_{u}, f_{u}} \sum_{u \in U}\left(\pi_{u} K_{u}-\left(c_{u}+\nu_{u}\right)\right) f_{u}
$$

under constraints (9), (10) and (21).

It appears that for any given feasible frequency distribution, maximizing the profit of the airlines sector will lead to increase $\pi_{u}$ on each link. According to properties $(1-a)$ and $(1-b)$ of the demand functions, this will lead to a diminution of demand which will end when:

$$
K_{u} \cdot f_{u}=D_{u}(\underline{\pi}, \underline{f}) \quad u \in U
$$

Then, here it is considered that an efficient behavior for airlines will be to provide a supply no greater than the expected demand while all expected profitable demand should be satisfied. This leads to the equilibrium conditions:

$$
\phi_{u}=K_{u} \cdot f_{u}=D_{u}(\underline{\pi}, \underline{f}) \forall u \in U
$$

Considering the invertibility property of $\left(D_{u}, u \in U\right)$ with respect to $\left(\pi_{u}, u \in U\right)$, from (24), a bijective mapping $F$ from $\underline{\pi} \in R^{|U|}$ to $f \in R^{|U|}$ can be defined, so that:

$$
\underline{f}=\bar{F}(\underline{\pi}) \text { and } \underline{\phi}=K \cdot F(\underline{\pi})
$$

where $K=\operatorname{diag}\left\{K_{1}, \cdots, K_{|U|}\right\}$.

Then problem (7)-(10) is replaced by:

$$
\max _{\pi_{u}, u \in U} \sum_{u \in U}\left(\pi_{u} \cdot K_{u}-\left(c_{u}+\nu_{u}\right) \cdot\right) F_{u}(\underline{\pi})
$$

under

$$
\sum_{u \in U} L_{u} \cdot F_{u}(\underline{\pi}) \leq F_{U}
$$

and

$$
\pi_{u} \geq 0 \quad u \in U
$$

In this study we consider particularly the case in which $F$ is an affine function with respect to $\underline{\pi}$ such as:

$$
F(\underline{\pi})=\underline{f_{0}}-\Phi \cdot \underline{\pi}
$$

where $\Phi$ is a square matrix of dimensions $|U|$.

Then we get for the airlines sector the following linear quadratic optimization problem:

$$
\max _{\pi_{u}, u \in U} \underline{\pi}^{t} Q \underline{\pi}+\underline{P}^{t} \underline{\pi}+R \text { with } \underline{S}^{t} \underline{\pi} \geq \operatorname{Tand} \underline{\pi} \in(R+)^{|U|}
$$

where

$$
\begin{aligned}
& Q=K \Phi, \underline{P}=-\left(\Phi^{t}(\underline{c}+\underline{\nu})+K \underline{f_{0}}\right), \\
& R=(\underline{c}+\underline{\nu})^{t} \underline{f_{0}}, \underline{S}=\Phi \underline{L} \text { and } T=\underline{L} \underline{\Phi} \Phi \underline{\pi}
\end{aligned}
$$


Problem (30) is a linear quadratic problem which can be numerically solved easily by using algorithms such as $[22,23]$. However, adopting some rather credible assumptions, the solution of this problem can be turned analytic. For instance, taking into account relations $(1-\mathrm{a})$ :

$$
\Phi_{u u}>0 \forall u \in U, \Phi_{u v} \leq 0 \text { and }\left|\Phi_{u v}\right|<<\Phi_{u u} \forall u, v \in U, u \neq v
$$

and considering that $K$ is a diagonal matrix, it is expected that matrix $Q^{s}$ given by $Q^{s}=\left(Q+Q^{t}\right) / 2$ will be a definite positive symmetric matrix. Here it is also useful to Pricing
schemes for air
traffic services assume that:

$$
\Phi_{u u} L_{u}>\sum_{v \neq u} \Phi_{u v} L_{v} \forall u \in U
$$

so that $\underline{S}$ is a positive vector and that:

$$
F_{U} \geq \underline{L^{t}} \underline{f_{0}}
$$

Then $\underline{S}$ is a positive vector and $T$ is a positive scalar see (27), (29), so that the capacity constraint vanishes and the solution of problem (30) is given by:

$$
\underline{\pi} *=-\frac{1}{2}\left(Q^{s}\right)^{-1} \underline{P}
$$

or

$$
\underline{\pi} *=\frac{1}{2}\left(Q^{s}\right)^{-1}\left(\Phi^{t}(\underline{c}+\underline{v})+K \underline{f_{0}}\right)
$$

\section{Optimal mean ticket rates by the airlines sector}

Now assuming that the whole airlines sector adopts as reference price a mean ticket rate per flown hour $p$, so ticket rates are defined such as:

$$
\pi_{u}=L_{u} p \quad u \in U
$$

problem (26)-(28) can be rewritten as a scalar optimization problem:

$$
\max _{p \in R^{+}} g(\underline{\nu}, p) \text { under } h(p) \leq F_{U}
$$

where:

$$
g(\underline{\nu}, p)=p \cdot \underline{L^{t}} K \cdot F(\underline{L} \cdot p) \text { and } h(p)=\underline{L^{t}} \cdot F(\underline{L} \cdot p)
$$

In the case in which $F$ is an affine function, see relation (29), and that $\Phi$ is such as (32) then the optimization criteria of problem (38) becomes:

$$
\max _{p \in R^{+}} \alpha p^{2}+\beta p+\gamma, \quad p \geq \delta
$$

with

$$
\alpha=-\underline{L} \underline{L}^{t} K \Phi \underline{L}, \quad \beta=-\left(\underline{L^{t}} K \underline{f_{0}}+(\underline{c}+\underline{\nu})^{t} \Phi \underline{L}\right)
$$

and 


$$
\gamma=-(\underline{c}+\underline{\nu})^{t} \cdot f_{0}, \quad \delta=\left(\underline{L^{t}} f_{0}-F_{U}\right) /\left(\underline{L^{t}} \Phi \underline{L}\right)
$$

Since $\alpha$ is expected to have a negative value, the general solution of this problem is given by:

$$
p^{*}=\max \left\{\delta, \frac{-\beta}{2 \alpha}\right\}
$$

98

Or

$$
p^{*}=\max \left\{\frac{\left(\underline{L}^{t} f_{0}-F_{u}\right)}{\underline{L}^{t} \Phi \underline{L}}, \frac{\left(\underline{L^{t} K} \underline{f_{0}}+(\underline{c}+\underline{\nu})^{t} \Phi \underline{L}\right)}{2 \underline{L^{t}} K \Phi \underline{L}}\right\}
$$

Then, the ATS fees will have an influence on the transportation fares chosen by the airlines sector if the maximum of (44) is given by the second term, this can be written:

$$
\underline{\nu}^{t} \Phi \underline{L} \geq \varepsilon
$$

with

$$
\varepsilon=\underline{L}^{t}\left(2 \frac{\underline{L}^{t} K \Phi \underline{L}}{\underline{L^{t}} \Phi \underline{L}}-K\right) \underline{f_{0}}-2 \frac{\underline{L}^{t} K \Phi \underline{L}}{\underline{L^{t}} \Phi \underline{L}} F_{U}-\underline{c}^{t} \Phi \underline{L}
$$

The optimal ticket rate $p^{*}$ is given in that case by:

$$
p^{*}=p_{0}+\underline{\rho}^{t} \cdot \underline{\nu}
$$

with

$$
p_{0}=\frac{\underline{L}^{t}}{2 \underline{L^{t} K \Phi \underline{L}}}\left(K \underline{f_{0}}+\Phi^{t} \underline{c}\right) \text { and } \underline{\rho}=\frac{(\Phi \underline{L})^{t}}{2 \underline{L^{t} K \Phi \underline{L}}}
$$

and the optimal frequencies and expected demand are given by:

$$
\underline{f}^{*}(\underline{\nu})=\underline{g_{0}^{*}}-G_{\nu} \cdot \underline{\nu} \text { and } \underline{\phi}^{*}(\nu)=K f^{*}(\underline{\nu})
$$

with

$$
\underline{g_{0}^{*}}=\underline{f_{0}}-p_{0} \Phi \underline{L} \text { and } G_{\nu}=\Phi \underline{L} \underline{\rho}{ }^{t}
$$

The optimal frequency and expected demand are such as:

$$
f^{*}=\left(1-\frac{\Phi \underline{L} \underline{L}^{t}}{\underline{L} \Phi \underline{L}}\right) \underline{f}_{0}+\frac{\Phi \underline{L}}{\underline{L}} F_{U} \text { and } \underline{\phi}^{*}=K f^{*}
$$

\section{Pricing of ATS with a public supplier}

Here it is supposed that the market conditions for international connections are already established so that their economic return for the airlines sector and the ATS provider are already known. According to relations (12)-(15), when considering that condition (45) holds, the problem of optimization solved by the public ATS provider to choose a level for the ATS fees such as demand is promoted can be rewritten under the form: 


$$
\min _{\underline{v}} \underline{K}^{t} G_{\nu} \underline{\nu}
$$

with the constraints:

$$
\begin{gathered}
\underline{v}^{t} N_{T} \underline{v}+\underline{M}_{T} \underline{v}+z_{T} \geq 0 \\
\underline{v}^{t} N_{A} \underline{v}+\underline{M}_{A} \underline{v}+z_{A} \geq 0 \\
\nu_{u} \geq 0 \quad \forall u \in U
\end{gathered}
$$

where

$$
\begin{gathered}
N_{T}=-G_{\nu}+\alpha_{\mathrm{int}} \lambda_{U} G_{v}^{t} K \underline{L} \underline{\rho}^{t} \\
M_{T}=\left(1-\alpha_{\min } \lambda_{U} \underline{\rho} \underline{L}^{t} K\right) \underline{g}_{0}^{*}+G_{v}^{t}\left(\underline{\sigma}+\alpha_{\mathrm{int}} \lambda_{U} K \underline{L} \underline{p}_{0}\right) \\
z_{T}=\sum_{e \in E}\left(\left(v_{e}-\sigma_{e}\right) f_{e}+\alpha_{e x t} \lambda_{E} \phi_{e} \pi_{e}\right)-C_{A T S}^{F}-\alpha_{\mathrm{int}} \lambda_{U} \underline{L}^{t} K \underline{g}_{0}^{*}-\underline{\sigma}^{t} \underline{g}_{0}^{*}-R_{A T S}^{\min } \\
N_{A}=\left(1-\left(1-\lambda_{U}\right) \underline{\rho}^{t} \underline{L}^{t} K\right) G_{\nu} \\
M_{A}=\left(1-\lambda_{U}\right) \underline{L} \underline{L}^{t} K\left(\underline{g}_{0}^{*} \underline{\rho}^{t}-p_{0} K G_{\nu}\right)+\underline{c}^{t} G_{\nu}-\underline{g}_{0}^{*} \\
z_{A}=\left(\left(1-\lambda_{U}\right) p_{0} \underline{L} \underline{\underline{L}} K-\underline{c}^{t}\right) \cdot \underline{g}_{0}^{*}+\mu \cdot \sum_{e \in E}\left(\pi_{e}\left(1-\lambda_{E}\right) \phi_{e}\right. \\
\left.-\left(c_{e}+v_{e}\right) f_{e}\right)-C_{A L S}^{F}-R_{A L S}^{\min }
\end{gathered}
$$

In general, $N_{T}$ is definite negative, whereas $N_{A}$ is definite negative. Solution methods can be found in [21,22].

Figure 2 illustrates the two-dimensional case (two air links operated in a single sector of air traffic control) (Figure 3):

The feasible region is represented by the area which is the intersection of the profitability areas of the airlines sector (ALS) and of the ATS (ATC). Here the demand level lines are straight lines parallel to $\Delta$, whereas the optimal solution is at point $A$.

In the case in which the solution of (52)-(55) does not satisfy condition (45), the volume of demand is fixed and given by (51). Then the optimum problem reduces to finding a feasible solution to the linear set of constraints with respect to $\underline{\underline{ }}$ given by:

$$
\begin{gathered}
\underline{f}^{* t} \cdot \underline{\nu}+\left(\alpha_{\mathrm{int}} \lambda_{U} \sum_{u \in U} \phi_{u}^{*} \pi_{u}^{*}-\underline{\sigma}^{t} \cdot \underline{f}^{*}+\sum_{e \in E}\left(\left(v_{e}-\sigma_{e}\right) f_{e}+\alpha_{e x t} \lambda_{E} \sum_{e \in E} \phi_{e} \pi_{e}\right)-C_{A T S}^{F}-R_{A T S}^{\min }\right) \geq 0 \\
\underline{f}^{* t} \cdot \underline{v}+\left(\sum_{u \in U} \underline{p}^{*} L_{u}\left(1-\lambda_{U}\right) \cdot \phi_{u}^{*}-\underline{c}^{t} \cdot \underline{f}^{*}+\mu \cdot \sum_{e \in E}\left(\pi_{e}\left(1-\lambda_{E}\right) \phi_{e}\right.\right. \\
\left.\left.-\left(c_{e}+v_{e}\right) f_{e}\right)-C_{A L S}^{F}-R_{A L S}^{\min }\right) \geq 0 \\
\underline{\nu}^{t} \Phi \underline{L} \leq \varepsilon
\end{gathered}
$$

with (55) where $p^{*}$ is given by (44), $f^{*}$ and $\underline{\phi}^{*}$ are given by (51). 


\section{$\mathrm{ACI}$ \\ 17,1}

100

\section{Figure 2.}

Solution for public ATS supplier (bidimensional case).

\section{Figure 3.}

Solution for private ATS supplier (bidimensional case).
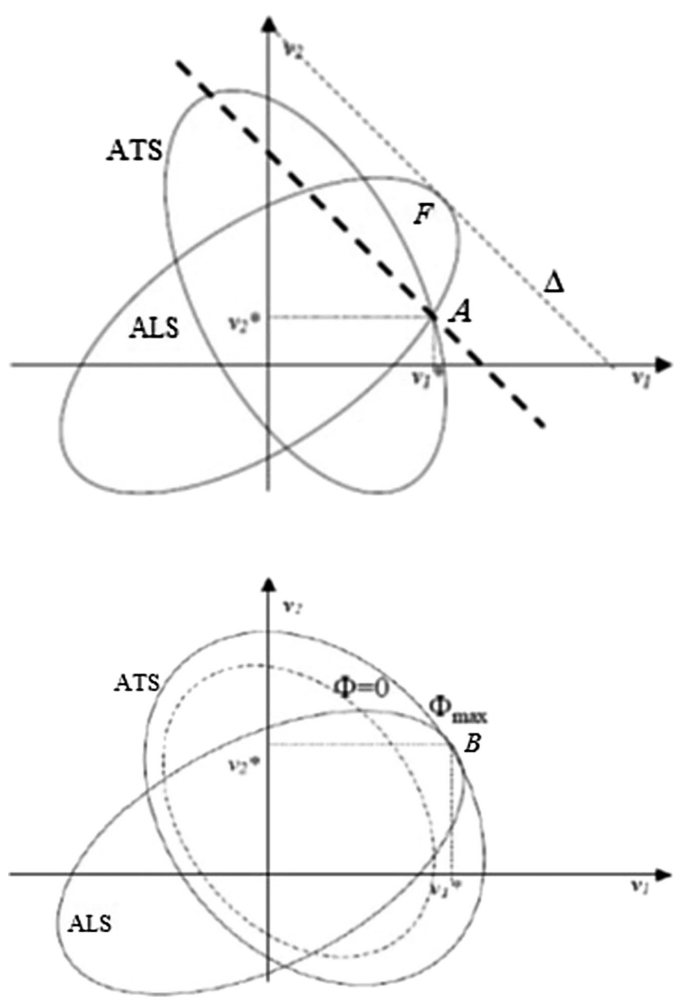

Now considering that ATS fees are established on a flown time basis, an ATS rate per flown hour $v$ can be introduced such as:

$$
\nu_{u}=L_{u} v u \in U
$$

which is solution when (45) is satisfied of the scalar optimization problem:

$$
\min _{v \in R^{+}} \underline{K}^{t} G_{\nu} \underline{L} v
$$

under the constraints:

$$
\begin{gathered}
\left(\underline{L}^{t} N_{T} \underline{L}\right) v^{2}+\left(\underline{M}_{T} \underline{L}\right) v+z_{T} \geq 0 \\
\left(\underline{L}^{t} N_{A} \underline{L}\right) v^{2}+\left(\underline{M}_{A} \underline{L}\right) v+z_{A} \geq 0 \\
\left(\underline{L}^{t} \Phi \underline{L}\right) v \geq \varepsilon
\end{gathered}
$$

If the feasible set associated to constraints (65)-(67) is empty, the ATS supplier can adopt the solution of the scalar optimization problem:

$$
\max _{v \in R^{+}} f^{* t} \cdot \underline{\nu}
$$

under the constraint 


$$
\underline{v}^{t} \Phi \underline{L} \leq \varepsilon
$$

with (44) where $p^{*}$ is given by (51), $f_{-}^{*}$ and $\underline{\phi}^{*}$ are given by (51).
Pricing

schemes for air traffic services

\section{Pricing of ATS with a private supplier}

According to relations (17)-(19), considering that condition (35) holds, the problem of optimization solved by the public ATS provider can be rewritten under the form:

$$
\max _{\underline{v} \in\left(R^{+}\right)|U|} \underline{v}^{t} N_{T} \underline{v}+\underline{M}_{T} \underline{v}+z_{T}
$$

under the constraints:

$$
\begin{gathered}
\underline{v}^{t} N_{A} \underline{v}+\underline{M}_{A} \underline{v}+z_{A} \geq 0 \\
\left(\underline{L}^{t} \Phi \underline{L}\right) v \geq \varepsilon
\end{gathered}
$$

Here also, solution methods can be found in [21,22].

The feasible region is represented by the area which is the intersection of the profitability areas of the airlines sector (ALS) and of the ATS (ATC). Here the demand level lines are not represented, the profit level lines are parameterized by the profit level, whereas the optimal solution is at point $B$. If the feasible set associated to constraints (57) and (58) is empty, the ATS supplier can adopt the solution of the scalar optimization problem (68), (69) with (55) where $p^{*}$ is given by (44) while $f^{*}$ and $\phi^{*}$ are given by (51).

Here also, if we are interested in the ATS rate per flown hour $v$, the problem of optimization solved by the public ATS provider can be rewritten under the scalar form:

$$
\max _{\underline{v} \in\left(R^{+}\right)^{|U|}}\left(\underline{L}^{t} N_{T} \underline{L}\right) v^{2}+\left(\underline{M_{T}} \underline{L}\right) v+z_{T}
$$

under the constraints:

$$
\begin{gathered}
\left(\underline{L}^{t} N_{A} \underline{L}\right) v^{2}+\left(\underline{M_{A}} \underline{L}\right) v+z_{A} \geq 0 \\
\left(\underline{L^{t}} \Phi \underline{L}\right) v \geq \varepsilon
\end{gathered}
$$

\section{General solution algorithm}

The problem considered in Section 5 with a linear criterion and quadratic constraints can be considered to be a special case of a non convex linear program with LMI constraints [23] such as:

$$
\min \underline{c}^{t} \underline{z} \text { under } M^{1}(z) \geq 0 \text { and, } \quad M^{2}(z) \geq 0
$$

where $c \in R^{m}$ is given and where:

$$
M^{j}(z)=M_{0}^{j}+\sum_{i=1}^{m} z_{i} M_{i}^{j} j=1,2
$$

$M_{i}^{j} j, i=0$ to $m, j=1,2$ are symmetric matrices. 


$$
\max w \text { with } \underline{v}^{t} N_{T} \underline{v}+\underline{M}_{T}^{t} \underline{v}+z_{T}-w \geq 0
$$

where $w$ is the level of the objective function.

General non convex problem (76) and (77) can be solved through an ellipsoid algorithm which has been developed in the field of LMI's [24]. At start it is supposed that an ellipsoid $E_{0}$ in $R^{\mathrm{m}}$ contains the feasible set and hence the optimal solution. A cutting plane crossing the center $z_{c}(0)$ of this ellipsoid is chosen so that the optimal solution lies in one of the half spaces of $R^{\mathrm{m}}$ given by:

$$
\left\{z \in R^{m}: \underline{\nu}(0)^{t}\left(\underline{z}-\underline{z}_{c}(0)\right) \leq 0\right\}
$$

where $\nu(0)$ is a non zero vector of $R^{m}$. Then an ellipsoid $E_{1}$ with minimum generalized volume and containing the half ellipsoid given by:

$$
E_{0} \cap\left\{z \in R^{m}: \underline{\nu}(0)^{t}\left(\underline{z}-\underline{z}_{c}(0)\right)\right\}
$$

is constructed. The size of this ellipsoid is smaller than the one of the previous ellipsoid and contains the solution. This process can be repeated until a required accuracy is achieved.

Given an ellipsoid $E_{k}$ given by:

$$
\left\{z \in R^{m}:\left(\underline{z}-\underline{z}_{c}(k)\right)^{t} A_{k}^{-1}\left(\underline{z}-\underline{z}_{c}(k)\right) \leq 1\right\}
$$

where $A_{k}$ is a symmetric definite positive matrix, the minimum volume ellipsoid $E_{k+1}$ containing the half ellipsoid:

$$
E_{0} \cap\left\{z \in R^{m}: \underline{\nu}(k)^{t}\left(\underline{z}-\underline{z}_{c}(k)\right) \leq 0\right\}
$$

is given by

$$
\left\{z \in R^{m}:\left(\underline{z}-\underline{z_{c}}(k+1)\right)^{t} A_{k}^{-1}\left(\underline{z}-\underline{z}_{c}(k+1)\right) \leq 1\right\}
$$

where:

$$
\begin{gathered}
\underline{z}_{c}(k+1)=\underline{z_{c}}(k)-\frac{1}{m+1} A_{k} \underline{w_{k}} \\
\underline{w_{k}}=\underline{v_{k}} / \sqrt{\underline{\nu_{k}^{t}} A_{k} \underline{v_{k}}}
\end{gathered}
$$

and

$$
A_{k+1}=\frac{m^{2}}{m^{2}-1}\left(A_{k}-\frac{2}{m+1} A_{k} \underline{w}_{k} \underline{w}_{k}^{t} A_{k}\right)
$$

Then considering at step $k$ a point $\underline{\nu_{k}}$ in $R^{m}$, two cases can be considered:

- either $M^{1}\left(\underline{y}_{k}\right) \geq 0$ and $M^{2}\left(\underline{y}_{k}\right) \leq 0$, in that case one takes $\underline{\nu}_{k}=\underline{c}$ and the half space:

$$
\left\{z \in R^{m}: \underline{\nu}_{k}^{t}\left(\underline{z}-\underline{y}_{k}\right)>0\right\}
$$

can be deleted since there $\underline{c}^{t} z=\underline{c}^{t} y_{k}$ and points $\underline{z}$ cannot be solution of the optimization problem. 
- or $M^{1}\left(\underline{y}_{k}\right)<0$ or $M^{2}\left(\underline{y}_{k}\right)>0$, there exists a non zero vector $u$ of $R^{\mathrm{n}}$ such that according to the case:

$$
\underline{u}^{t}\left(M_{0}^{1}+\sum_{i=1}^{m} \underline{z}_{i} M_{i}^{1}\right)<0 \text { or } \underline{u}^{t}\left(M_{0}^{2}+\sum_{i=1}^{m} \underline{z}_{i} M_{i}^{j}\right)>0
$$

then choosing:

$$
\underline{\nu}_{k i}=-\underline{u}^{t} M_{i}^{j} \underline{u}, \quad i=1 \text { to } m
$$

we have for every $\underline{z} \in R^{m}$ such that $\underline{\nu}_{k}^{t}\left(\underline{z}-\underline{y}_{k}\right) \geq 0$ :

$$
\underline{u}^{t} M^{1}(z) \underline{u}=\underline{u}^{t} M^{1}\left(\underline{y}_{k}\right) \underline{u}-\underline{\nu}_{k}^{t}\left(\underline{z}-\underline{y}_{k}\right)<0 \text { or } \underline{u}^{t} M^{2}(z) \underline{u}=\underline{u}^{t} M^{2}\left(\underline{y}_{k}\right) \underline{u}-\underline{\nu}_{k}^{t}\left(\underline{z}-\underline{y}_{k}\right)>0
$$

The feasible set will be in the half space:

$$
\left\{z \in R^{m}: \underline{\nu}_{k}^{t}\left(\underline{z}-\underline{y}_{k}(0)\right)<0\right\}
$$

and $\underline{\nu}_{k}$ allows to define the cutting plane at point $\underline{\nu}_{k}$. Then the whole process is repeated until the size of the ellipsoid becomes sufficiently small to insure accuracy of the solution. It can be shown [5] that convergence is exponential.

\section{Numerical application}

To illustrate the proposed approach, we consider the case of the air traffic area represented in Figure 4.

Table 1 provide the adopted values for the main parameters of the considered air traffic network:

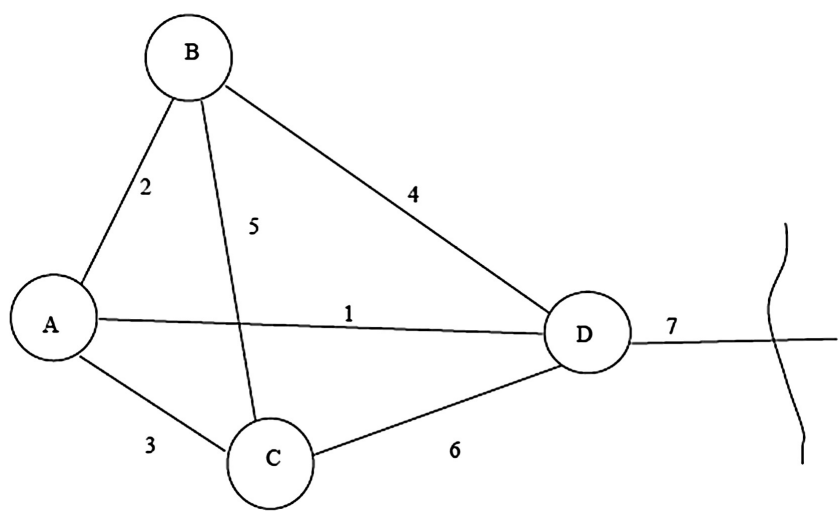

\begin{tabular}{|c|c|c|c|c|c|c|c|c|}
\hline Link $u$ & 1 & 2 & 3 & 4 & 5 & 6 & 7 & \\
\hline$L_{u}$ (hours) & 2 & 1 & 1 & 2 & 1 & 1 & 2 & $\begin{array}{r}\text { Table } 1 . \\
\text { Adopted values for air }\end{array}$ \\
\hline$K_{u}$ (seats) & 100 & 100 & 100 & 100 & 100 & 100 & 200 & links delays, capacity \\
\hline $\mathrm{c}_{\mathrm{u} \text { (Euros) }}$ & 8000 & 4000 & 4000 & 8000 & 4000 & 4000 & 16000 & and costs. \\
\hline
\end{tabular}

Figure 4. The considered air traffic network. 
$\mathrm{ACI}$

17,1

104

Here demand is supposed to depend only on the average price of tickets. Relation (92) display the average daily demand for each link.

$$
\begin{gathered}
D(,)=\left[\begin{array}{c}
100 \\
80 \\
60 \\
100 \\
40 \\
70 \\
120
\end{array}\right]-\left[\begin{array}{ccccccc}
0.010 & 0.000 & 0.000 & 0.000 & 0.000 & 0.000 & 0.0000 \\
0.000 & 0.010 & 0.000 & 0.000 & 0.000 & 0.000 & 0.000 \\
0.000 & 0.000 & 0.013 & 0.000 & 0.000 & 0.000 & 0.000 \\
0.000 & 0.000 & 0.000 & 0.012 & 0.000 & 0.000 & 0.000 \\
0.000 & 0.000 & 0.000 & 0.000 & 0.0012 & 0.000 & 0.000 \\
0.000 & 0.000 & 0.000 & 0.000 & 0.000 & 0.010 & 0.000 \\
0.000 & 0.000 & 0.000 & 0.000 & 0.000 & 0.000 & 0.007
\end{array}\right]\left[\begin{array}{c}
\pi_{1} \\
\pi_{2} \\
\pi_{3} \\
\pi_{4} \\
\pi_{5} \\
\pi_{6} \\
\pi_{7}
\end{array}\right] \\
C_{A T S}^{F}=85000, R_{A T S}^{\min }=130000, C_{A L S}^{F}=1020000, R_{A L S}^{\min }=1550000
\end{gathered}
$$

Minimum returns for the ATS and the ALS have been taken equal to 1550000 Euros and 130 000 Euros respectively while fixed costs for ATS and ALS have been taken equal to 85000 Euros and 1020000 Euros respectively.

Tables 2 and 3 displays the obtained results for different values of $\sigma_{0}$ and $\lambda$ given in $\%$, unit for the ATS service rate $v$ is Euros per flight hour, units for mean tickets prices $\pi_{i}$ are Euros. Decreases of fuel costs and other expenses which are included in $C_{A L S}^{F}$ and $C_{A T S}^{F}$ have been considered allowing to introduce higher levels for $R_{A L S}^{\min }$ and $R_{A T S}^{\min }$. With the following values, $C_{A T S}^{F}=82000$ Euros, $R_{A T S}^{\min }=132000$ Euros, $C_{A L S}^{F}=1000000$ Euros, $R_{A L S}^{\min }=1560000$ Euros, the resulting pricings are given in Table 4 and 5.

\section{Discussion of the results}

In all the considered numerical cases, the adopted solution algorithm (Section 9) produced the optimal solution in a reduced number of iterations. This has allowed to consider large range of variations for the average ATS costs $(\sigma)$ and for airlines tax rate $(\lambda)$ while sensitivity analysis with respect to other relevant parameters could be performed. A global view of prices at the network level is obtained which is of interest for both the ATS and the airlines sector. In the considered demand structure (relation 92) no competition has been introduced between destinations but this situation could have been tackled easily by the proposed approach. In

\begin{tabular}{cccccccccc}
\hline$\sigma_{0}$ & $\lambda$ & $\nu$ & $\pi_{1}$ & $\pi_{2}$ & $\pi_{3}$ & $\pi_{4}$ & $\pi_{5}$ & $\pi_{6}$ & $\pi_{7}$ \\
\hline 20 & 0.10 & 108.06 & 216.12 & 108.06 & 108.06 & 216.12 & 108.06 & 108.06 & 216.12 \\
& 0.15 & 105.14 & 210.28 & 105.14 & 105.14 & 210.28 & 105.14 & 105.14 & 210.28 \\
& 0.20 & 102.22 & 204.44 & 102.22 & 102.22 & 204.44 & 102.22 & 102.22 & 204.44 \\
& 0.25 & 99.3 & 198.6 & 99.3 & 99.3 & 198.6 & 99.3 & 99.3 & 198.6 \\
30 & 0.10 & 118 & 236 & 118 & 118 & 236 & 118 & 118 & 236 \\
& 0.15 & 115 & 230 & 115 & 115 & 230 & 115 & 115 & 230 \\
& 0.20 & 112 & 224 & 112 & 112 & 224 & 112 & 112 & 224 \\
40 & 0.25 & 109 & 218 & 109 & 109 & 218 & 109 & 109 & 218 \\
& 0.10 & 128 & 256 & 128 & 128 & 256 & 128 & 128 & 256 \\
& 0.15 & 125 & 250 & 125 & 125 & 250 & 125 & 125 & 250 \\
& 0.20 & 122 & 244 & 122 & 122 & 244 & 122 & 122 & 244 \\
50 & 0.25 & 119 & 238 & 119 & 119 & 238 & 119 & 119 & 238 \\
& 0.10 & 138 & 276 & 138 & 138 & 276 & 138 & 138 & 276 \\
& 0.15 & 135 & 270 & 135 & 135 & 270 & 135 & 135 & 270 \\
& 0.20 & 132 & 264 & 132 & 132 & 264 & 132 & 132 & 264 \\
& 0.25 & 129 & 258 & 129 & 129 & 258 & 129 & 129 & 258 \\
\hline
\end{tabular}

Table 2.
Pricing results for the public ATS case. 


\begin{tabular}{|c|c|c|c|c|c|c|c|c|c|c|}
\hline$\sigma_{0}$ & $\lambda$ & $\nu$ & $\pi_{1}$ & $\pi_{2}$ & $\pi_{3}$ & $\pi_{4}$ & $\pi_{5}$ & $\pi_{6}$ & $\pi_{7}$ & \\
\hline \multirow[t]{4}{*}{20} & 0.10 & 136.5 & 273 & 136.5 & 136.5 & 273 & 136.5 & 136.5 & 273 & \\
\hline & 0.15 & 142 & 284 & 142 & 142 & 284 & 142 & 142 & 284 & \\
\hline & 0.20 & 149 & 298 & 149 & 149 & 298 & 149 & 149 & 298 & \\
\hline & 0.25 & 155 & 310 & 155 & 155 & 310 & 155 & 155 & 310 & \\
\hline \multirow[t]{4}{*}{30} & 0.10 & 136.5 & 273 & 136.5 & 136.5 & 273 & 136.5 & 136.5 & 273 & \\
\hline & 0.15 & 142 & 284 & 142 & 142 & 284 & 142 & 142 & 284 & \\
\hline & 0.20 & 149 & 298 & 149 & 149 & 298 & 149 & 149 & 298 & \\
\hline & 0.25 & 155 & 310 & 155 & 155 & 310 & 155 & 155 & 310 & \\
\hline \multirow[t]{4}{*}{40} & 0.10 & 136.5 & 273 & 136.5 & 136.5 & 273 & 136.5 & 136.5 & 273 & \\
\hline & 0.15 & 142 & 284 & 142 & 142 & 284 & 142 & 142 & 284 & \\
\hline & 0.20 & 149 & 298 & 149 & 149 & 298 & 149 & 149 & 298 & \\
\hline & 0.25 & 155 & 310 & 155 & 155 & 310 & 155 & 155 & 310 & \\
\hline \multirow[t]{4}{*}{50} & 0.10 & 136.5 & 273 & 136.5 & 136.5 & 273 & 136.5 & 136.5 & 273 & Table 3. \\
\hline & 0.15 & 142 & 284 & 142 & 142 & 284 & 142 & 142 & 284 & Pricing results for the \\
\hline & 0.20 & 149 & 298 & 149 & 149 & 298 & 149 & 149 & 298 & private ATS case in \\
\hline & 0.25 & 155 & 310 & 155 & 155 & 310 & 155 & 155 & 310 & Europe. \\
\hline
\end{tabular}

\begin{tabular}{rrrrrrrrrr}
\hline$\sigma_{0}$ & \multicolumn{1}{c}{$\boldsymbol{\lambda}$} & \multicolumn{1}{c}{$\boldsymbol{\nu}$} & $\pi_{1}$ & $\pi_{2}$ & $\pi_{3}$ & $\pi_{4}$ & $\pi_{5}$ & $\pi_{6}$ & $\pi_{7}$ \\
\hline 20 & 0.10 & 107 & 214 & 107 & 107 & 214 & 107 & 107 & 214 \\
& 0.15 & 104 & 208 & 104 & 104 & 208 & 104 & 104 & 208 \\
& 0.20 & 102 & 204 & 102 & 102 & 204 & 102 & 102 & 204 \\
& 0.25 & 99 & 198 & 99 & 99 & 198 & 99 & 99 & 198 \\
30 & 0.10 & 117 & 234 & 117 & 117 & 234 & 117 & 117 & 234 \\
& 0.15 & 114 & 228 & 114 & 114 & 228 & 114 & 114 & 228 \\
& 0.20 & 112 & 224 & 112 & 112 & 224 & 112 & 112 & 224 \\
& 0.25 & 109 & 218 & 109 & 109 & 218 & 109 & 109 & 218 \\
40 & 0.10 & 127 & 254 & 127 & 127 & 254 & 127 & 127 & 254 \\
& 0.15 & 124 & 248 & 124 & 124 & 248 & 124 & 124 & 248 \\
& 0.20 & 122 & 244 & 122 & 122 & 244 & 122 & 122 & 244 \\
& 0.25 & 119 & 238 & 119 & 119 & 238 & 119 & 119 & 238 \\
50 & 0.10 & 137 & 274 & 137 & 137 & 274 & 137 & 137 & 274 \\
& 0.15 & 134 & 268 & 134 & 134 & 268 & 134 & 134 & 268 \\
& 0.20 & 132 & 264 & 132 & 132 & 264 & 132 & 132 & 264 \\
& 0.25 & 129 & 258 & 129 & 129 & 258 & 129 & 129 & 258
\end{tabular}

Table 4.

Pricing results for the public ATS case.

\begin{tabular}{cccccccccc}
\hline$\sigma_{0}$ & $\lambda$ & $\nu$ & $\pi_{1}$ & $\pi_{2}$ & $\pi_{3}$ & $\pi_{4}$ & $\pi_{5}$ & $\pi_{6}$ & $\pi_{7}$ \\
\hline 20 & 0.10 & 121 & 242 & 121 & 121 & 242 & 121 & 121 & 242 \\
& 0.15 & 127 & 254 & 127 & 127 & 254 & 127 & 127 & 254 \\
& 0.20 & 134 & 268 & 134 & 134 & 268 & 134 & 134 & 268 \\
& 0.25 & 140 & 280 & 140 & 140 & 280 & 140 & 140 & 280 \\
30 & 0.10 & 121 & 242 & 121 & 121 & 242 & 121 & 121 & 242 \\
& 0.15 & 127 & 254 & 127 & 127 & 254 & 127 & 127 & 254 \\
& 0.20 & 134 & 268 & 134 & 134 & 268 & 134 & 134 & 268 \\
& 0.25 & 140 & 280 & 140 & 140 & 280 & 140 & 140 & 280 \\
40 & 0.10 & 121 & 242 & 121 & 121 & 242 & 121 & 121 & 242 \\
& 0.15 & 127 & 254 & 127 & 127 & 254 & 127 & 127 & 254 \\
& 0.20 & 134 & 268 & 134 & 134 & 268 & 134 & 134 & 268 \\
& 0.25 & 140 & 280 & 140 & 140 & 280 & 140 & 140 & 280 \\
50 & 0.10 & 121 & 242 & 121 & 121 & 242 & 121 & 121 & 242 \\
& 0.15 & 127 & 254 & 127 & 127 & 254 & 127 & 127 & 254 \\
& 0.20 & 134 & 268 & 134 & 134 & 268 & 134 & 134 & 268 \\
& 0.25 & 140 & 280 & 140 & 140 & 280 & 140 & 140 & 280
\end{tabular}

Table 5.

Pricing results for the private ATS case in Europe. 
the considered numerical case developed in this section, public ATS provides in general

better results for travellers (lower ticket fares) than private ATS, airlines results remain stable (lower fares compensated by higher demand) while ATS results are lower in the public case. According to tax levels, fares can be modified (+ or -) up to $15 \%$ and demand can be modified (+ or -) up to $10 \%$. However, adopting different values for the parameters as well as considering different network structures, could lead to different conclusions.

\section{Conclusion}

In this article we addressed the complex problem of ATS pricing at network level by integrating within a new multilevel framework the behavior of the different involved economic agents. Then, it has been possible to take into account the reactivity of supply by the airline companies and of demand by users as a result of the variation of ATS charges and air tickets prices.

The proposed framework allows in particular to tackle the issue of having either a public or a private ATS provider by introducing differentiated objectives depending of the nature of the ATS provider and leading to different optimization problems. This has resulted in the formulation of two different multilevel programming problems with a common lower level problem associated with the profit maximization behavior of the airline sector. This lower problem has been tackled on a multidimensional basis. It has been shown that when mean spatial rates are considered, it is possible under mild assumptions to solve analytically this problem.

The higher problems associated to the behavior of a private or a public ATS provider, result in quadratic constrained optimization problems which can be easily solved numerically using a specialized version of the ellipsoid algorithm.

The proposed approach allows for different sets of cost and demand parameters, the extensive comparison of the optimal solutions in terms of expected aircraft/passengers flows for the whole network and in terms of economic returns for the ATS provider and the airline sector.

The complexity of the considered issue has been tackled by designing a multilevel solution approach which produces, through the successive resolution of reduced numerical problems, a sound basis for decision by public authorities to pursue efficiency and fairness at network level for ATS pricing.

\section{References}

[1] M.W. Tretheway, Cost Allocation Principles for ATC, Conference on Air Traffic Economics, Belgrade (2009).

[2] United States Government Accountability Office, Characteristics and performances of selected international air navigation service providers and lessons learned from their commercialisation, United States Government Accountability Office, Report to Congressional requesters, July 1-34 (2005).

[3] A. A. Oumarou and F. Mora-Camino, Pricing in air transportation systems: a multilevel approach, XIV Congreso Panamericano de Ingenieria de Transito y Transporte, September 20-23, Las Palmas de Gran Canaria, Spain, 2006.

[4] R. Guettaf, M. Larbani, F. Mora-Camino, Pricing of ATC/ATM Services with a Private Provider, VIII SITRAER, São Paulo, 2009.

[5] R. Guettaf, C. Mancel, M. Larbani, F. Mora-Camino, Pricing of ATC/ATM services through bilevel programming approaches, J. Braz. Air Trans. Res. Soc. (2010).

[6] J.F. Bard, Practical Bi-level Optimization: Algorithms and Applications. Kluwer Book Series: Non Convex Optimization and its Applications 30 (1998). 
[7] S. Dempe, Foundations of Bi-Level Programming, Kluwer Academie Publishers, Dordrecht, 2002.

[8] G. Savard, J. Gauvin, The steepest descent direction for the nonlinear bilevel programming problem, Oper. Res. Lett. 15 (1994) 265-272.

[9] Marianne Raffarin, Congestion in European airspace a pricing solution?, J. Trans. Econ. Policy 38 (1) (2004) 109-125.

[10] A. Ranieri, L. Castelli, Pricing schemes based on air navigation service charges to reduce en-route ATFM delays, Third International Conference on Research in Air Transportation, 2008, Fairfax, VA.

[11] T. Bolic, L. Castelli, D. Rigonat, Peak-load pricing for the European air traffic management system using modulation of en-route charges, Eur. J. Trans. Infrastruct. Res. 17 (1) (2017) 136-152.

[12] R. Jovanovic, V. Tosic, M. Cangalovic, M. Stanojevic, Anticipatory modulation of air navigation charges to balance the use of airspace network capacities, in: Transportation Research Part A, Policy and Practice, Elsevier, 2014, pp. 84-99.

[13] M. Labbé, A. Violin, Bilevel programming and price setting problem, in: Annals OR, Springer, 2016, pp. 141-169.

[14] A. Violin, M. Labbe, L. Castelli, En route charges for ANSP revenue Maximization, 4th International Conference on Research in Air Transport, 2010. Budapest.

[15] L. Castelli, M. Labbe, A. Violin, A Network Pricing Formulation for the Revenue Maximization of European Air Navigation Service Providers, ORP3 Meeting, Cadiz, Sept. 13-17 (2011).

[16] ICAO, Manual on Air Navigation Services Economics, Doc 9161, 2013 Edition.

[17] P. Holder, Airline Operation Costs, prepared for: Managing Aircraft Maintenance Costs Conference, Brussels 22 (2003).

[18] ICAO, Tariffs for Airports and Air Navigation Services, 2010 Edition.

[19] Eurocontrol, Guidelines for the Implementation of Single European Sky Legislation, the Military Released Issue, ed. 1.0, 14/07/2009.

[20] United States Government Accountability Office, Assigning Air Traffic Control Costs to Users Elements of FAA's (2010).

[21] R.M. Freund, Solution Methods for Quadratic Optimization, Massachusetts Institute of Technology, 2004.

[22] E.M. Gertz, S.J. Wright, Object-oriented software for quadratic programming, ACM Trans. Math. Software 29 (1) (2003) 58-81 2003.

[23] S. Boyd, L. El Ghaoui, E. Feron, V. Balakrishnan, Linear Matrix Inequalities in Systems and Control Theory, SIAM Studies in Applied Mathematics, Philadelphia, 1994.

[24] B. Dane, Improved, ellipsoid algorithm for LMI feasibility problems, Int. J. Control, Automation Syst. 7 (6) (2009) 1015-1019.

\section{Corresponding author}

Félix Mora-Camino can be contacted at: moracamino@hotmail.fr

For instructions on how to order reprints of this article, please visit our website:

www.emeraldgrouppublishing.com/licensing/reprints.htm

Or contact us for further details: permissions@emeraldinsight.com 